\title{
Study on EGR Control Strategy for Vehicle Engine Based on Simulation
}

\author{
Liu Yongfeng \\ Beijing University of Civil Engineering and Architecture \\ Beijing, China. \\ e-mail: liuyongfeng@bucea.edu.cn
}

\author{
Qinhui Zhou \\ Beijing University of Civil Engineering and Architecture \\ Beijing, China.
}

\begin{abstract}
EGR strategy in diesel engine the calculation model using GT-POWER is built. the thesis uses simulation software GT-POWER to establish the calculation model of $4 \mathrm{JB} 1$ intake system of turbocharged diesel engine with EGR system and the results agree well. According to the simulation results, it can predict the main performance indicators of the diesel engine when it works at the maximum torque condition, which includes A/F, IMEP, Maximum Pressure, and Intake Pressure and so on. Then after controlling the volume of gas by modifying the EGR valve, it can find out the regulation how EGR effects the engine's emissions, torque, power and the inlet temperature of turbo. Referring to the simulation results, it includes from four aspects : EGR can significantly improve the quality of emission gas; NOx and PM have a trade-off relationship; applying higher EGR rate in heavy load can lead to the low torque and power less; the inlet temperature of turbo increases with the augment of EGR rate. In the experiment, the inlet oxygen concentration method is used to determine the EGR rate, and to realize it, the oxygen concentration detection module is also successful designed. Then a close-loop control strategy is proposed based on the EGR rate. It proves it is feasible to guide the ECU programmer development by the simulation results of engine control strategy. It is also can be referred by other engine control systems development.
\end{abstract}

Keywords-Terms-Simulation; GT-POWER; EGR; Control Strategies.

\section{INTRODUCTION}

Electronic control technology can improve the performance of diesel engines, currently, electronic controlled fuel injection system, such as the high pressure common rail and electronic controlled unit pump is only applied to light and middle duty diesel engine. In the heavy duty diesel engine, it is a main way to use electronic governor. The noxious emissions in exhaust gases from internal combustion engines of vehicles are one of the most remarkable compositions of air pollution. Because environmental protection enjoys more and more populations these days, to reduce the noxious atmospheric emissions, in which nitrogen oxides (NOx) and particulate matter (PM) play a major role, seems just in the direction of engines'

\author{
Jianwei Yang, \\ Beijing University of Civil Engineering and Architecture \\ Beijing, China.
}
Aihua Zhu
Beijing University of Civil Engineering and Architecture Beijing, China.

development. The technology Exhaust Gas Recirculation (EGR) is effective to solve the problem of emissions and the EGR valve is required to be installed in the engine according to critical emission standard.

In this paper, simulation-based diesel engine EGR system control strategy. Firstly, the simulation software GTPOWER platform to establish common-rail with a supercharged engine EGR system model, a single element of EGR on engine performance were studied, and the simulation results to develop a corresponding EGR control strategy. Later, in Matlab / Simulink platform, EGR system control logic to build guide ECU microcontroller-based programming. Finally, the experimental method validation control strategy and control logic is correct.

\section{EGR SYSTEM CONTROL STRATEGY DESIGN}

\section{A. Basic Simulation Model}

Conventional EGR control logic, there are two, one is open-loop EGR control, one is the EGR valve opening based on the closed-loop feedback control. Open-ring control logic in the EGR, ECU according to engine speed, load, temperature, air flow condition signals, calibration of the EGR rate with pulse spectrum, calculated in line with the best conditions when EGR rate, and drives a control high precision pulse control type (PWM) vacuum control valve (EVR), and then indirectly controlled by the EGR vacuum regulator valve diaphragm valve chamber of the vacuum, then change the EGR valve opening degree, adjust the EGR rate. The greater the duty cycle, equivalent to the electromagnetic coils is relatively longer, the greater the vacuum diaphragm chamber, EGR valve opening height greater access to more gas in the cylinder, EGR rate. Therefore, ECU as long as the control exerted on the EVR solenoid valve duty cycle on the pulse voltage, you can achieve variable control of EGR rate. The open-loop control EGR, EGR rate can only be pre-set, can not detect and control the engine operating conditions the actual EGR rate. In addition, the vacuum tube still lag effect, it is the shortcomings of this control method GT-POWER platform built 4JB1 engine model, shown in Figure 1. Model 1 is for the intake system, exhaust system 
model 2 and 3 for the exhaust gas recirculation loop model, box 4 turbo system within the model, box 5 within the engine block model.

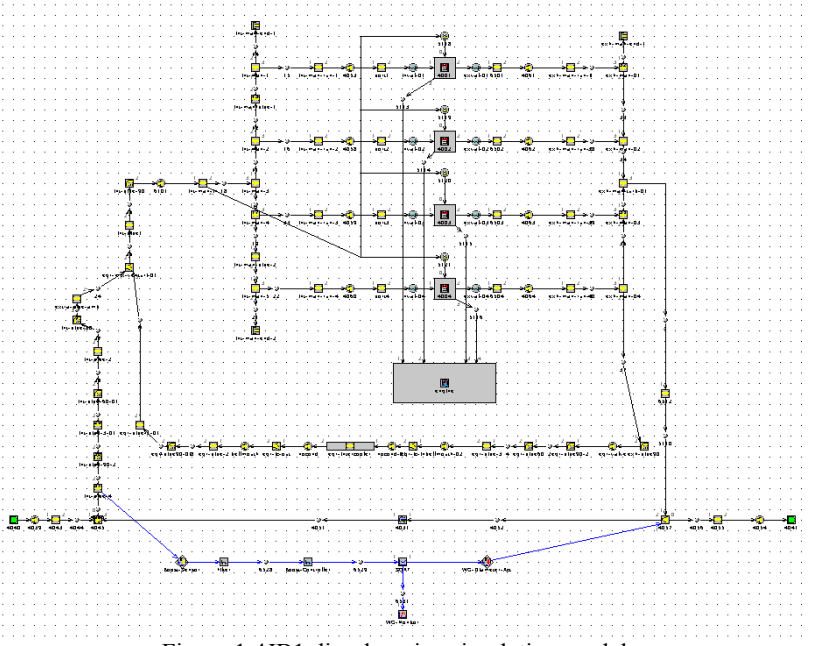

Figure $14 \mathrm{JB} 1$ diesel engine simulation model

\section{B. EGR control strategy}

Diesel engine combined with practical work and the dynamic process of economic needs and the EGR on the simulated performance and emissions of diesel engine rule, the macro-control policy to develop EGR as follows:

1) the idle, the combustion temperature is not high, NOx emissions is not, in order to make the engine idle stability, and should cut off the EGR.

2) the engine is started, to make the engine a smooth start and stable operation, need to cut off the EGR.

3) The engine cooling water temperature is low, combustion instability, and the combustion temperature is lower, you need to cut off the EGR. With the cooling water temperature, EGR increases.

4) When it is low-speed cruise in a slight acceleration, due to less fuel injection, combustion volume becomes unstable, you can use a small amount of EGR, to reduce NOx emissions, while ensuring that the driver of good.

5) Medium engine load, NOx emissions are high at this time a large EGR rate should be used to reduce NOx emissions. With the increase of load, EGR rate can be increased accordingly.

6) Since a large load, EGR due to torque loss and power loss will increase, so the engine requires more power, in order to ensure good power; can use less or no EGR.

\section{$C$ EGR rate based on optimization}

EGR rate optimization principle is essentially the same level of particulate emissions on the basis of lower NOx. EGR can be seen from the performance prediction, in a small load conditions, EGR on engine power and economy has little effect at high load conditions, EGR will cause the engine power and economy deteriorated to some extent. Therefore, should determine the optimal EGR reduces NOx, smoke and the engine power to increase the level of damage and requirement of large dynamic loads considering the point of view, a large selection of small load EGR rate, the selection of small high-load EGR rate, EGR rate of full load 0 . Low load conditions the particles, NOx as the EGR rate factors. When oxygen is plentiful due to the small air-fuel ratio larger load, the introduction of EGR, particulate essentially the same, so a large EGR rate should be adopted. Particles with the heavy load because the increase of EGR rate of the rapid increase in inflection point exists, it should adopt the small EGR rate, EGR rate, and that the inflection point in this. Taking into account the atmospheric temperature and pressure change should be using smaller EGR rate. In this simulation study was $1300 \mathrm{r} / \mathrm{min}$, 2000r/min, 2400r/min and 4 different torque different EGR rates, a total of 70 points predicted NOx and particulate emissions, and as a basis for EGR rate optimization. Optimized EGR rate is in Table 1. When taking into account the idle condition the exhaust temperature is lower, in order to prevent condensation of water and EGR NOx generated after acid corrosion on the negative impact of diesel engine production, idling condition without EGR. Air-fuel ratio when the diesel engine low load, without EGR. NOx emissions at low speed are not a major problem, so this design program is used when speed is lower than $1200 \mathrm{r} / \mathrm{min}$ without EGR.

TABLE I. OPTIMIZED EGR RATE（\%

\begin{tabular}{|c|c|c|c|c|}
\hline Tpeed & 40(N.m) & $80($ N.m $)$ & $120($ N.m $)$ & $160($ N.m $)$ \\
\hline $1300(\mathrm{r} / \mathrm{min})$ & 8.9 & 8.2 & 10.1 & 6.1 \\
\hline $2000(\mathrm{r} / \mathrm{min})$ & 15.5 & 11.3 & 12.3 & 9.6 \\
\hline $2400(\mathrm{r} / \mathrm{min})$ & 17.4 & 15.8 & 11.6 & 5.4 \\
\hline
\end{tabular}

III SimUlATION AND EXPERIMENT COMPARATIVE ANALYSIS

Figure 2 (a) EGR and torque diagram and Figure 2 (b) EGR and power relations can be seen that, in a small load, torque and power independent of the impact of EGR rate, a large load torque Moment and power began to decline with the increase of EGR rate, and the load the greater the drop more. Figure 2 (c) can be seen, NOx concentration with the increase of EGR rate decreases monotonically. Relative to low load, high load conditions, the same inhibitory effect of EGR rate on the NOx is more evident. Figure 2 (d) EGR and smoke can be seen in the diagram, a small load, the smoke with the EGR less affected., The heavy load, the small EGR, the impact of smoke by the EGR is not large, but when the EGR rate is greater than a certain critical point, the smoke increased with the rapid increase in EGR. The critical point increases as the load to move in the direction of small EGR 
rate. When experiments of 2000r/min EGR on engine performance were studied and simulation speed obtained in the performance of EGR's impact on the laws of the engine matched the speed proved the correctness of the simulation results.

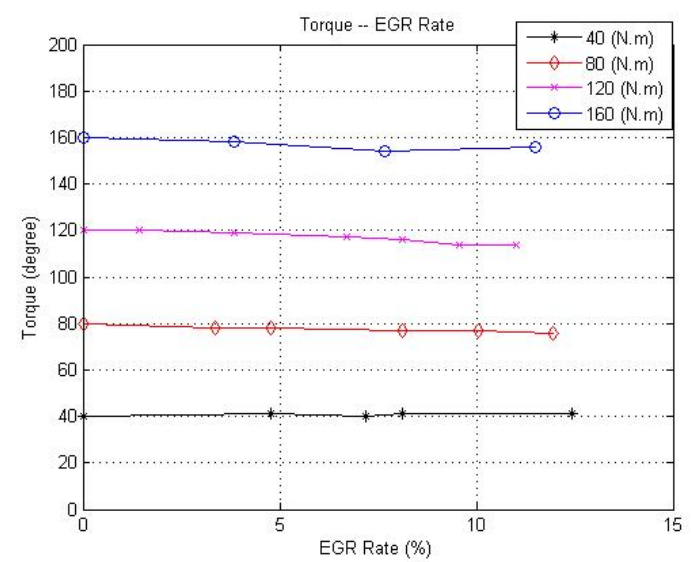

Figure 2 (a) Torque-EGR rate

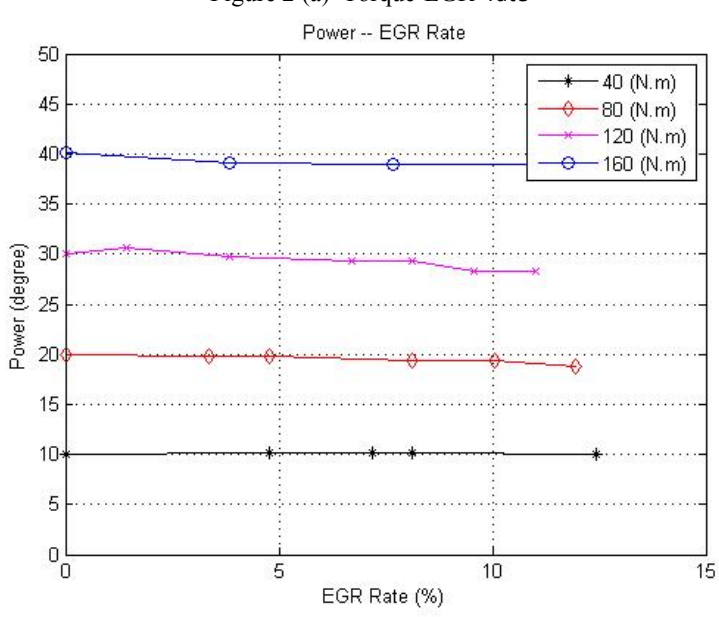

Figure 2(b) Power-EGR rate

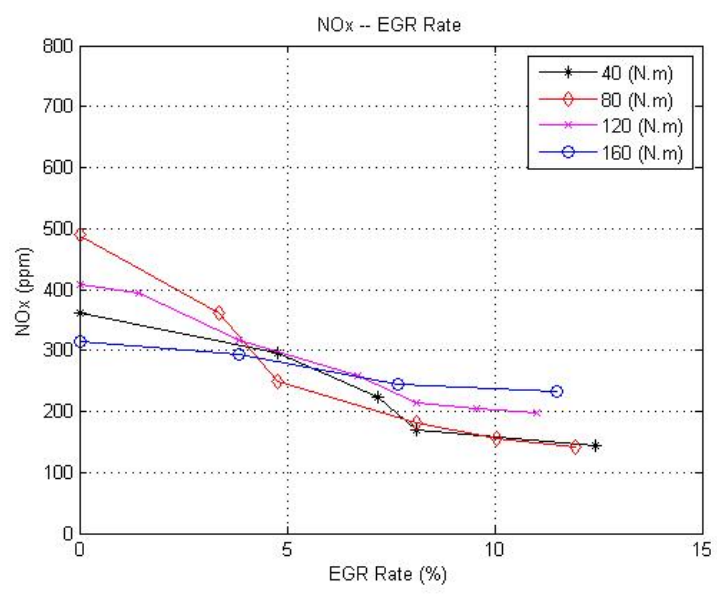

Figure 2(c) NOx-EGR rate

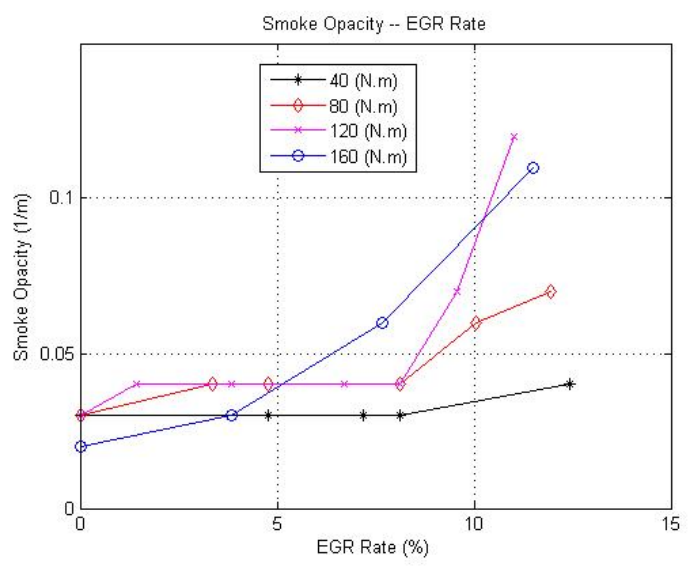

Figure 2(d) Smoke-EGR rate

\section{IV . CONCLUSIONS}

Through the simulation and experiment of the EGR control strategy we know as follows:

(1) When the engine is idle, the combustion temperature is not high, NOx emissions is not, in order to make the engine idle stability, cut off the EGR.

(2) When the engine starts, in order to start the engine a smooth and stable operation, need to cut off the EGR; engine cooling water temperature is low, combustion instability, and the combustion temperature is lower, you need to cut off the EGR. With the cooling water temperature, increasing EGR; in minor or low-speed cruising speed, because less fuel injection, combustion volume becomes unstable, you can use a small amount of EGR, to reduce NOx emissions, while ensuring that the driver of good ; medium engine load, NOx emissions are high at this time a large EGR rate should be used to reduce NOx emissions. With the increase of load, EGR rate can be increased accordingly.

(3) When the engines require high power, high speed, in order to ensure better power, fuel injection quantity is large at this time, NOx emissions resultant reduced, and therefore can EGR or less without using EGR; in the intake air temperature is low, the combustion temperature inside the cylinder will be reduced, this time should reduce the amount of exhaust gas recirculation to the combustion process can be a good manner.

\section{ACKNOWLEDGMENT}

This project has been supported by Beijing Natural Science Foundation (3102011) and Beijing University of Civil Engineering and Architecture Ph.D foundation (101001604) and Funding Project for Academic Human Resources Development in Institutions of Higher Learning 
Under the Jurisdiction of Beijing Municipality (PHR(IHLB)

201008370,201106125).

\section{REFERENCES}

[1] Alain Maiboom, Xavier Tauzia, Jean-Franc-ois He'tet, Experimental study of various effects of exhaust gas recirculation (EGR) on combustion and emissions of an automotive direct injection diesel engine, Energy, 33(2008)22-34

[2] CHEN Juan, WANG Jun. Study on Electro-hydraulic Injection Timing Actuator's Response Speed for Diesel Engine [J]. Vehicle Engine. 2006 (1):37 39 (in Chinese)

[3] LI Tao. Development of Dynamic Acquisition System of Injection Ahead Angle in vehicular Diesel Engine[D]. Beijing: Beijing Institute of Technology, 2004 (in Chinese)

[4] Liu Yongfeng, Mesh Generation and Dynamic Mesh Management for KIVA-3V , Journal of Beijing Institute of Technology[J],2009.2,5,41-45

[5] Huang Maoyang, Zhang Jing, and Zai Yujian, "Research on the Performance of High-speed Solenoid for Common-Rail Type Injector", Chinese Journal of Scientific Instrument, Bei Jing, pp.29-31, Aug. 2004.

[6] G.M.Bianchi, P.Pelloni, F.Filicori, and G.Vannini, "Optimization of the Solenoid Valve Behavior in Common-Rail Injection System", Sae2000-01-2042, SAE Publications Group, USA, June 19-22. 2000, pp. 1-4.

[7] Viktor Szente etc, "Computational and experimental Investigation on Solenoid Valve Dynamics", IEEE/ASME International Conference on Advanced Intelligent Mechatronics Proceedings, July. 2001, pp. 618623.

[8] Takashi Kajima, Member, IEEE, and Yoshihisa Kawamura, "Development of a High-Speed Solenoid Valve: Investigation of Solenoids", IEEE Transaction on Industrial Electronics, Feb. 1995, pp $1-8$.

[9] N.C. Cheung, K.W. Lim and M.F. Rahman, "Modelling a linear andlimited travel solenoid", IEEE Proc. on Industrial Electronics Societjannual general meeting, ECON93, Hawaii, vol. 3, pp 15551563,November, 1993.

[10] Huang Weigang, Wang Xuyong, Wang Xianzheng, and Zhong Tingxiu, "Investigation on the Mechanism of Switching Characteristic of High-Speed On-Off Solenoid Valve", Journal of Shang Hai Jiao Tong university, Dec.1998, pp. 38-41. 